\title{
A Unified Approach to Integration Theory
}

\author{
Mangatiana A. Robdera* \\ Private Bag 0022, Department of Mathematics, University of Botswana, Gaborone. \\ * Corresponding author. Tel.: +267 75302045; email: robdera@yahoo.com \\ Manuscript submitted March 15, 2018; accepted October 14, 2018. \\ doi: 10.17706/ijapm.2019.9.1.21-28
}

\begin{abstract}
We investigate the common features and the resemblance of the central parts of the different existing integration theories to obtain a more unified approach to the notion of integral. Our approach gives a presentation of the integral that does not require the development of measure theory.
\end{abstract}

Key words: Riemann, Lebesgue-Bochner, Henstock-Kurzweil, McShane, Bartle integrals.

\section{Introduction}

Somewhere about the time a student reaches certain level of mathematical analysis, he/she may perhaps begin to wonder how many more forms the concept of integral can assume. It is desirable to have all the different but related approaches to integration put into fewer packages, so that it would not be necessary to do almost the same thing over, and over again for slightly different kinds of integral.

Modern integration theory is the culmination of centuries of refinements and extensions of ideas dating back to the Greeks. The first attempt to provide the theory a firm foundation was started by Riemann in the mid nineteenth century. His approach successfully gave the expected answers to many already solved problems and led to useful results for many new problems. However, the Riemann integral (R-integral) is quickly found to be inadequate for more advanced mathematics. The R-integral does not interact well with taking limits of sequences of functions. To correct such an important deficiency, Lebesgue developed his integral around the turn of the twentieth century, that later became the official integral adopted by most mathematicians. As powerful as it is, the Lebesgue integral (L-integral) has also its own limitations. The L-integral does not fare well with the Fundamental Theorem of Calculus. Some improper integrals do not exist as L-integrals. In 1912, Denjoy introduced the theory of gauge integral (HK-integral). Such a theory was given its more elegant definition by Kurzweil in 1957, and later on developed by Henstock. McShane showed that the L-integral can also be presented as gauge integral and gets a definition of the McShane integral [1].

As more and more techniques of analysis were extended to normed vector spaces, the needs to come up with even more general approaches to the integration becomes obvious. The Bochner integral extends the L-integral to strongly measurable vector-valued functions (see [2], [3]). The Pettis integral also extends the L-integral to vector-valued functions by exploiting duality [4]. The Bartle-Dunford-Schwartz integral [5] integrates scalar functions with respect to $\sigma$-additive vector measure. Integration of vector valued functions with respect to vector valued measures has been investigated by some authors (see e.g. [6]).

Recently, the author has introduced a novel approach to integration. Ideas and results related to such an approach can be seen in [7]-[12], Our aim in this paper is to give an overview of such new approach by gathering the main ideas from the above listed references. We give a treatment of the integration theory 
that is not only more unified but is also elegant and easily understood.

\section{Brief Review of the Classical Integrals}

We shall denote by $I(a, b)$ any interval with end points $a<b$ and by $\ell(I(a, b))=b-a$ its length. A partition of $[a, b]$ is a collection $P=\left\{I\left(a_{i}, b_{i}\right): i=1, \ldots, n\right\}$ of finitely many pairwise disjoint subintervals $I\left(a_{i}, b_{i}\right)$ whose union is $[a, b]$. A partition $P$ is said to be tagged if for each $i$, a number $t_{i}$ is chosen from $I\left(a_{i}, b_{i}\right) . P$ is then called a tagged partition. Given a function $f:[a, b] \rightarrow \mathbb{R}$, and a tagged partition $P$ of $[a, b]$, the Riemann sum of $f$ at $P$ is the number $S_{f}(P):=\sum_{1=1}^{n} f\left(t_{i}\right) \ell\left(I\left(a_{i}, b_{i}\right)\right)$.

A number $A \in \mathbb{R}$ is the R-integral of a function $f:[a, b] \rightarrow \mathbb{R}$ if for every $\varepsilon>0$, there is a constant $\delta_{\varepsilon}>0$ such that if $P$ is any tagged partition of $[a, b]$ satisfying $\left[a_{i}, b_{i}\right] \subset\left(t_{i}-\delta_{\varepsilon}, t_{i}+\delta_{\varepsilon}\right),\left|S_{f}(P)-A\right|<\varepsilon$.

A more general definition of the integral is obtained if one allows $\delta_{\varepsilon}$ to be any continuous positive function $\delta:[a, b] \rightarrow(0, \infty)$ called a gauge function. A tagged partition $P$ of $[a, b]$ is said to be $\delta$-fine if $0<b_{i}-a_{i}<\delta\left(t_{i}\right)$ for $i=1, \ldots, n$. The existence of a $\delta$-fine partition is guaranteed by the Nested Intervals Theorem.

$A$ number $A \in \mathbb{R}$ is the HK-integral of a function $f:[a, b] \rightarrow \mathbb{R}$ if for every $\varepsilon>0$, there is a function $\delta:[a, b] \rightarrow(0, \infty)$ such that if $P$ is any $\delta$-fine tagged partition of $[a, b],\left|S_{f}(P)-A\right|<\varepsilon$.

If we drop the requirement that for $\left(I\left(a_{i}, b_{i}\right), t_{i}\right) \in P, t_{i} \in I\left(a_{i}, b_{i}\right)$, then the above defined number $A$ is exactly the McShane integral of $f$ over the interval $[a, b]$.

Given a non-empty set $\Omega$, and $\Sigma$ a $\sigma$-algebra of subsets of $\Omega$, a set function $\mu: \Sigma \rightarrow[0, \infty]$ is called a measure if it satisfies: $\mu(\emptyset)=0$ and $\mu\left(\cup_{k=1}^{\infty} E_{k}\right)=\sum_{k=1}^{\infty} \mu\left(E_{k}\right)$ for every collection $\left\{E_{k}: k \in \mathbb{N}\right\}$ of pairwise disjoint sets in $\Sigma$. The L-integral is first defined for non-negative simple functions, that is, functions of the form $s=\sum_{k} a_{k} 1_{S_{k}}$, where $a_{k} \geq 0, \quad S_{k} \in \Sigma$, and $\mu\left(S_{k}\right)<\infty$ whenever $a_{k} \neq 0$ : $\int_{\Omega} s d \mu=\sum_{k} a_{k} \mu\left(S_{k}\right)$. Then for non-negative functions on $\Omega$, one defines $\int_{\Omega} f d \mu=\sup \left\{\int_{\Omega} f d \mu: 0 \leq s \leq\right.$ $f, s$ simple $\}$. A function $f$ on $\Omega$ is measurable provided $\{\omega: a \leq f(\omega)<b\} \in \Sigma$ for all $a, b$. For every measurable function $f$ on $\Omega$, one can write $f=f^{+}-f^{-}$where $f^{+}:=f \vee 0$ and $f^{-}:=-f \wedge 0$. one then says that:

The L-integral of a measurable function $f: \Omega \rightarrow \mathbb{R}$ exists if $\min \left\{\int_{\Omega} f^{+} d \mu, \int_{\Omega} f^{-} d \mu\right\}<\infty$. And in this case, the L-integral of $f$ is given by $\int_{\Omega} f d \mu=\int_{\Omega} f^{+} d \mu-\int_{\Omega} f^{-} d \mu$.

The L-integral can be defined on domain spaces that do not require any topological notion. It has nice properties that made analysis involving integrals possible on a firm logical footing in ways that it has not been before. The L-integral allows us to work on nice spaces, namely, the $L^{P}$-spaces; that are in fact Banach spaces. By contrast, there is no known natural topology for the space of HK-integrable functions. For more details on the different approaches to classical integration theories, the reader is referred to [13].

\section{Elements of Integration}

\subsection{Integrators}

Let $\Sigma$ be a semiring of subsets of a set $\Omega$, and $V$ a normed vector space. By an integrator, we mean an additive set function $\mu: \Sigma \rightarrow V$ satisfying: $\mu(\varnothing)=0, \max \{\|\mu(A)\|,\|\mu(B)\|\} \leq\|\mu(A \cup B)\| \leq\|\mu(A)\|+$ $\|\mu(B)\|$ for disjoint $A, B$ in $\Sigma$ such that $A \cup B \in \Sigma$. The pair $(\Omega, \mu)$ shall be called an integrator space.

The length function is an integrator of the semiring of subintervals. A measure is a non-negative real valued integrator defined on a $\sigma$-algebra. An outer measure defines an integrator on the power set of a set. 
If $A \subset \Omega$, a finite family $\left\{E_{i} \in \Sigma: i \in I\right\}$ is said to be a $\Sigma$-cover of $A$ if $A \subset \bigcup_{i \in I} E_{i}$. The norm-variation of an integrator $\mu: \Sigma \rightarrow V$ is defined to be the extended real-valued set function $\|\mu\|: 2^{\Omega} \rightarrow[0,+\infty]$ defined by $\|\mu\|(A):=\inf \left\{\sum_{i \in I}\left\|\mu\left(E_{i}\right)\right\|\right\}$ where the infinimum is taken over all $\Sigma$-covers of the set $A$.

\subsection{Subpartitions}

Let $\Sigma$ be a semiring of subsets of a set $\Omega$, and $\mu: \Sigma \rightarrow V$ an integrator. We shall call a $\mu$-subpartition of $\Omega$ any finite subset $P$ of $\Sigma$ satisfying $\|\mu(I)\|<\infty$ for all $I \in P$ and $I \cap J=\emptyset$ for $I \neq J$ in $P$. The mesh of $P$ is defined to be $\|P\|=\max \{\mu(I): I \in P\}$. We denote $\cup P:=\cup_{I \in P} I$. We say that a $\mu$-subpartition $P$ is:

- tagged if for each $I \in P$, one associates a point $t_{I} \in I$ such that $t_{I} \neq t_{J}$ whenever $I \neq J$ in $P$.

- unconditionally tagged if for each $I \in P$, one associates $t_{I} \in U P$ such that $t_{I} \neq t_{J}$ if $I \neq J$ in $P$.

We denote by $\Pi(\Omega, \mu)$ (resp. $\widetilde{\Pi}(\Omega, \mu)$ ) the set of all tagged (resp. unconditionally tagged) $\mu$-subpartitions of $\Omega$. Clearly, we have $\Pi(\Omega, \mu) \subset \widetilde{\Pi}(\Omega, \mu)$.

Let $P, Q \in \widetilde{\Pi}(\Omega, \mu)$. Then $Q$ is said to be a refinement of $P$ (we write $Q>P$ ) if $\|Q\| \leq\|P\|$ and $\cup P \subset \cup Q$. The relation $>$ is transitive. If $P, Q \in \widetilde{\Pi}(\Omega, \mu)$, we denote $P \wedge Q:=\{I \backslash \cup Q, I \cap J, J \backslash P: I \in$ $P, J \in Q\}$. Clearly, $P \wedge Q \in \widetilde{\Pi}(\Omega, \mu), \quad P \wedge Q>P$ and $P \wedge Q>Q$. Thus, the relation $>$ has the upper bound property. We gather that both the sets $\Pi(\Omega, \mu)$ and $\widetilde{\Pi}(\Omega, \mu)$ are directed [14] by the binary relation $>$

\subsection{Riemann-Tensor-Sums}

Let $U, V$ and $W$ be normed spaces and $\tau: U \times V \rightarrow W$ is a continuous bilinear mapping such that for every $(u, v) \in U \times V$, the following inequality holds $\|u\|_{U} \leq \sup \left\{\|\tau(u, v)\|_{W}:\|v\|_{V} \leq 1\right\}$. We shall simply call such a mapping a tensor. For more details on tensor product, we refer the reader to [1]. For example:

1) The duality tensor: $\tau\left(v, v^{\prime}\right)=\left\langle v, v^{\prime}\right\rangle$ from $V \times V^{\prime} \rightarrow \mathbb{K}$ where $V^{\prime}$ is the dual of $V$, and $\mathbb{K}=\mathbb{R}$ or C.

2) If $V$ is a Hilbert space, the inner product: $\tau(u, v)=\langle u, v\rangle$ from $V \times V \rightarrow \mathbb{K}$,

3) The scaling tensor: $\tau(\alpha, v)=\alpha v$ from $\mathbb{K} \times V \rightarrow V$ (or $\tau(v, \alpha)=\alpha v$ from $V \times \mathbb{K} \rightarrow V$ ).

We define the Riemann-tensor-sum of a function $f: \Omega \rightarrow U$ at a $\mu$-subpartition $P$ to be the element $f_{\mu}(P):=\sum_{I \in P} \tau\left(f\left(t_{I}\right), \mu(I)\right)$ of $W$. Since $(\Pi(\Omega, \mu),>)$ (resp. $\left.(\widetilde{\Pi}(\Omega, \mu),>)\right)$ is a directed set, the mapping $P \mapsto f_{\mu}(P)$ is a net. We denote the net-limit by $\int_{\Omega} \tau(f, \mu):=\lim _{P} f_{\mu}(P)\left(\operatorname{resp} \int_{\Omega}^{\sim} \tau(f, \mu):=\lim _{\tilde{P}} f_{\mu}(\tilde{P})\right)$ where $P$ (resp. $\widetilde{P}$ ) runs through $\Pi(\Omega, \mu)$ (resp. $\widetilde{\Pi}(\Omega, \mu)$ ). See [14] for details on Moore-Smith limit.

\subsection{Definition of the Integral}

Definition 1. Let $U, V$ and $W$ be normed spaces, $\tau: U \times V \rightarrow W$ a tensor, $\Sigma$ a semiring of subsets of a nonempty set $\Omega$, and $\mu: \Sigma \rightarrow V$ an integrator. A function $f: \Omega \rightarrow U$ is integrable over $\Omega$ with respect to $\mu$ if the limit $\int_{\Omega} \tau(f, \mu)$ represents a vector in $W$. Such a vector is called the $\mu$-integral of $f$ over the set $\Omega$.

In other words, $f: \Omega \rightarrow U$ is $\mu$-integrable if there is $\int_{\Omega} \tau(f, \mu) \in W$ with the property that for every $\varepsilon>0$, there exists $P_{0} \in \Pi(\Omega, \mu)$ such that for every $P \in \Pi(\Omega, \mu), P>P_{0}$ we have $\left\|\int_{\Omega} \tau(f, \mu)-f_{\mu}(P)\right\|<$ $\varepsilon$.

The uniqueness of net-limit ensures us that there exists at most one vector $\int_{\Omega} \tau(f, \mu)$ that satisfies the property in the above Definition 1 . We also notice that being a limit operator, the integral is linear. Thus, the set of all $\mu$-integrable functions $f: \Omega \rightarrow U$ is a vector space which we shall denote by $\mathcal{J}(\Omega, \mu, U)$.

Let $A \subset \Omega$. Any $P \in \Pi(\Omega, \mu)$ can be refined to get a partition $P_{A}$ consisting of sets in $\Sigma$ that are either 
subsets of $A$ or disjoint from $A$. Then $\int_{\Omega} \tau\left(f 1_{A}, \mu\right)=\lim _{P_{A}} f_{\mu}\left(P_{A}\right)=\lim _{P_{A}} \sum_{I \in P} f\left(t_{I}\right) \mu(I \cap A)=\int_{A} \tau(f, \mu)$. If $A \cap B=\emptyset$, then every $R \in \Pi(A \cup B, \mu)$ is of the form $P \cup Q:=\{I \cup J: I \in P, J \in Q\}$ where $P \in \Pi(A, \mu)$ and $Q \in \Pi(B, \mu)$. It then follows that $f_{\mu}(R)=f_{\mu}(P)+f_{\mu}(Q)$. Thus, if $f: \Omega \rightarrow U$ is $\mu$-integrable over both $A$ and $B$, then $f$ is $\mu$-integrable over the union $A \cup B$ and $\int_{A \cup B} \tau(f, \mu)=\int_{A} \tau(f, \mu)+\int_{B} \tau(f, \mu)$.

If $\mu(A)=0$ then $f_{\mu}\left(P_{A}\right)=0$ and thus $\int_{\Omega} \tau(f, \mu)=0$. It follows that $\int_{\Omega} \tau(f, \mu)=\int_{\Omega} \tau(g, \mu)$ whenever $\|\mu\|(x \in \Omega: f(x) \neq g(x))=0$. We say that two functions $f, g: \Omega \rightarrow U$ are $\mu$-equivalent and we write $f \sim g$ if $\|\mu\|(x \in \Omega: f(x) \neq g(x))=0$. It is quickly seen that the relation $f \sim g$ is an equivalence relation on $\mathcal{J}(\Omega, \mu, U)$. We then denote by $I(\Omega, \mu, U)$ the quotient space $\mathcal{J}(\Omega, \mu, U) / \sim$.

Since partitions are subpartitions, our approach extends the R-integral and the HK-integral. The use of subpartition also allows our approach to cover the improper integral. Unlike the L-integral, our approach integrates functions that are not necessarily measurable on sets that are not necessarily measurable.

Definition 2. Let $U, V$ and $W$ be normed spaces, $\tau: U \times V \rightarrow W$ a tensor, $\Sigma$ a semiring of subsets of a nonempty set $\Omega$, and $\mu: \Sigma \rightarrow V$ an integrator. A function $f: \Omega \rightarrow U$ is unconditionally integrable over $\Omega$ with respect to $\mu$ if the limit $\int_{\Omega}^{\sim} \tau(f, \mu)$ represents a vector in $W$. Such a vector is then called the unconditional $\mu$-integral over the set $\Omega$.

We shall denote by $\tilde{J}(\Omega, \mu, U)$ the vector space of all unconditionally $\mu$-integrable functions and by $\tilde{I}(\Omega, \mu, U)$ the quotient space $\tilde{\mathcal{J}}(\Omega, \mu, U) / \sim$. It is clear that $\tilde{\mathcal{J}}(\Omega, \mu, U) \subset \mathcal{J}(\Omega, \mu, U)$.

\section{Topologizing the Space of Integrable Functions}

A topology can be easily defined on the space $\mathcal{J}(\Omega, \mu, U)$. For every $f: \Omega \rightarrow U$, we define:

- the $\mu$-variation of $f$ over $A$ to be $\operatorname{var}_{\mu}(f, A):=\sup \left\{\left\|f_{\mu}(P)\right\|: P \in \Pi(\Omega, \mu)\right\}$;

- the unconditional $\mu$-variation of $f$ over $A$ to be $\widetilde{\operatorname{var}}_{\mu}(f, A):=\sup \left\{\left\|f_{\mu}(P)\right\|: P \in \widetilde{\Pi}(\Omega, \mu)\right\}$.

We say that $f$ is of bounded $\mu$-variation (resp. unconditional $\mu$-variation) over $A$ if $\operatorname{var}_{\mu}(f, A)<\infty$ (resp. $\widetilde{\operatorname{var}}_{\mu}(f, A)<\infty$ ). If the vector spaces $U, V$ and $W$ are complete, we have the following interesting result.

Theorem 1. Let $U, V$ and $W$ be Banach spaces, $\tau: U \times V \rightarrow W$ a tensor, $\Sigma$ a semiring of subsets of a set $\Omega$, and $\mu: \Sigma \rightarrow V$ an integrator. If $A \subset \Omega$, $\|\mu\|(A)<\infty$, then $\mathcal{J}(A, \mu, U)$ (resp. $\tilde{\mathcal{J}}(A, \mu, U)$ ) is complete with respect to the seminorm $\operatorname{var}_{\mu}(f, A)$ (resp. $\widetilde{\operatorname{var}}_{\mu}(f, A)$ ). Hence, $I(A, \mu, U)$ (resp. $\tilde{I}(A, \mu, U)$ ) is a Banach space.

Proof. We prove the case of $\left(I(A, \mu, U), \operatorname{var}_{\mu}\right)$. Let $n \mapsto f_{n}$ be a Cauchy sequence in $I(A, \mu, U)$ with respect to $\operatorname{var}_{\mu}$. Fix $\varepsilon>0$, and let $N_{\varepsilon}>0$ such that for $m, n>N_{\varepsilon}$ in $\mathbb{N}$,

$$
\sup \left\{\left\|\left(f_{n}-f_{m}\right)_{\mu}(P)\right\|: P \in \Pi(A, \mu)\right\}<\varepsilon .
$$

For the subpartition $\{(A, a)\} \in \Pi(A, \mu)$, then for $m, n>N_{\varepsilon}$ in $\mathbb{N},\left\|\left(f_{n}-f_{m}\right)_{\mu}(A)\right\|_{W}<\|\mu\|(A) \varepsilon$. Since $\|\mu\|(A)<\infty$, we infer that the sequence $n \mapsto f_{n}(a)$ is Cauchy in $W$. Since $W$ is a Banach space, we can define $a \mapsto f(a)=\lim _{n \rightarrow \infty} f_{n}(a)$. Since $f_{n}, f_{m} \in I(A, \mu, U)$, there exist $P_{n}, P_{m} \in \Pi(A, \mu)$ such that

$$
\begin{gathered}
\left\|\left(f_{n}\right)_{\mu}(P)-\int_{\mathrm{A}} f_{n} d \mu\right\|<\varepsilon \text { whenever } P>P_{n}, \\
\left\|\left(f_{m}\right)_{\mu}(P)-\int_{\mathrm{A}} f_{m} d \mu\right\|<\varepsilon \text { whenever } P>P_{m} .
\end{gathered}
$$

Combining (4.1), (4.2) and (4.3), we have for $m, n>N_{\varepsilon}$ in $\mathbb{N}$ and for $P>P_{n} \vee P_{m}$, 


$$
\left\|\int_{\mathrm{A}} f_{m} d \mu-\int_{\mathrm{A}} f_{n} d \mu\right\| \leq\left\|\int_{\mathrm{A}} f_{m} d \mu-\left(f_{m}\right)_{\mu}(P)\right\|+\left\|\left(f_{n}-f_{m}\right)_{\mu}(P)\right\|+\left\|\left(f_{n}\right)_{\mu}(P)-\int_{\mathrm{A}} f_{n} d \mu\right\|<3 \varepsilon .
$$

Hence, the sequence $n \mapsto \int_{\mathrm{A}} f_{n} d \mu$ is Cauchy in $U$, and thus converges to, say $u \in U$. Now since for $a \in A \quad, \quad f(a)=\lim _{n \rightarrow \infty} f_{n}(a) \quad$ there exists $N_{a}>N_{\varepsilon}$ such that for $m, n>N_{\varepsilon},\left\|f_{n}(a)-f_{m}(a)\right\|_{U} \leq \varepsilon\|\mu\|(A)^{-1}$. It follows that for $P \in \Pi(\mathrm{A}, \Sigma, \mu)$, and for $m, n>\max \left\{N_{t_{I}}: I \in\right.$ $P\}=: N_{P}$, we have

$$
\left\|\left(f_{n}-f_{m}\right)_{\mu}(P)\right\| \leq \sum_{I \in P}\|\mu\|(A)\left\|f_{n}\left(t_{I}\right)-f_{m}\left(t_{I}\right)\right\|_{U}<\varepsilon
$$

If we let $m \rightarrow \infty$, we obtain $\left\|\left(f_{n}-f\right)_{\mu}(P)\right\| \leq \varepsilon$. Since $u=\lim _{n \rightarrow \infty} \int_{\mathrm{A}} f_{n} d \mu$, there exists $N>N_{P}$ such that $\left\|\int_{\mathrm{A}} f_{m} d \mu-u\right\|<\varepsilon$ whenever $m>N$. Thus for $n, m>N$,

$$
\left\|\left(f_{n}\right)_{\mu}(P)-u\right\| \leq\left\|\left(f_{n}-f\right)_{\mu}(P)\right\|+\left\|\left(f_{n}-f_{m}\right)_{\mu}(P)\right\|+\left\|\int_{\mathrm{A}} f_{m} d \mu-u\right\|<\varepsilon .
$$

Since $\varepsilon>0$ is arbitrary, this shows that $f \in \mathcal{J}(A, \mu, U)$ and that $\int_{\mathrm{A}} f d \mu=u$. The proof is complete.

\section{Cauchy Criterion for Integrability}

In this section, we give characterizations of $\mu$-integrability. Again, we fix normed vector spaces $U, V$ and $W$, a tensor $\tau: U \times V \rightarrow W$, a semiring $\Sigma$ of subsets of a given nonempty set $\Omega$, and an integrator $\mu: \Sigma \rightarrow V$.

Proposition 2. If $f \in \mathcal{J}(A, \Sigma, \mu, U)$ where $A$ is a given subset of $\Omega$, then for every $\varepsilon>0$, there exists $P_{0} \in \Pi(A, \mu)$ such that $\left\|f_{\mu}(Q)\right\|$ for every $Q \in \Pi(\mathrm{A}, \mu), Q \cap P_{0}=\emptyset$ and $\|Q\| \leq\left\|P_{0}\right\|$.

Proof. Fix $\varepsilon>0$. Let $P_{0} \in \Pi(A, \mu)$ such that $\left\|f_{\mu}(P)-\int_{A} \tau(f, \mu)\right\|<\varepsilon / 2$ for every $\in \Pi(A, \mu), P>P_{0}$. Fix such a $P$. Then for every $Q \in \Pi(A, \mu)$ such that $Q \cap P_{0}=\emptyset$ and $\|Q\| \leq\left\|P_{0}\right\|$, we have $P_{0} \vee Q \in$ $\Pi(A, \mu), P_{0} \vee Q>P_{0}$ and thus $\left\|f_{\mu}\left(P_{0} \vee Q\right)-\int_{A} \tau(f, \mu)\right\|<\varepsilon / 2$. It follows that

$$
\left\|f_{\mu}(Q)\right\| \leq\left\|f_{\mu}\left(P_{0} \vee Q\right)-f_{\mu}\left(P_{0} \vee Q\right)\right\| \leq\left\|f_{\mu}\left(P_{0} \vee Q\right)-\int_{A} \tau(f, \mu)\right\|+\left\|\int_{A} \tau(f, \mu)-f_{\mu}\left(P_{0}\right)\right\|<\varepsilon
$$

The proof is complete.

Similar result can be stated for $\tilde{\mathcal{J}}(A, \mu, U)$ and proved by simply using $\widetilde{\Pi}(A, \mu)$ in lieu of $\Pi(A, \mu)$. Given $P, Q \in \Pi(A, \mu)$, we shall denote $P \wedge Q:=\{I \cap J: I \in P, J \in Q\}$ and $P \triangle Q:=P \vee Q \backslash P \wedge Q$.

Definition 3. A function $f: \Omega \rightarrow U$ satisfy the Cauchy criterion for $\mu$-integrability (resp. for unconditional $\mu$-integrability) over a set $A \subset \Omega$ if for every $\varepsilon>0$, there exists $P_{0} \in \Pi(A, \mu)$ (resp. $P_{0} \in \widetilde{\Pi}(A, \mu)$ ) such that if $P, Q \in \Pi(A, \mu)$ (resp. $\widetilde{\Pi}(A, \mu)), P, Q>P_{0}$, we have $\left\|f_{\mu}(P \vee Q)-f_{\mu}(P \wedge Q)\right\|<\varepsilon$.

We notice that if $P, Q \in \Pi(A, \mu)$ (resp. $\widetilde{\Pi}(A, \mu)$ ), then $\left\|f_{\mu}(P \vee Q)-f_{\mu}(P \wedge Q)\right\|=\left\|f_{\mu}(P \triangle Q)\right\|$.

Proposition 4. A function $f: \Omega \rightarrow U$ satisfies the Cauchy criterion for $\mu$-integrability (resp. for unconditional $\mu$-integrability) over a $A \subset \Omega$ if and only if for every $\varepsilon>0$, there exists $P_{0} \in \Pi(A, \mu)$ (resp. $P_{0} \in \widetilde{\Pi}(A, \mu)$ ) such that $\left\|f_{\mu}(Q)\right\|<\varepsilon$ for every $Q \in \Pi(A, \mu), Q \cap P_{0}=\emptyset$.

The converse of Proposition 2. holds for the case where $U, V, W$ are complete. This follows from the well-known fact that for nets taking values in a Banach space, the Cauchy net condition is equivalent to the net convergence (see e.g. [14]). Clearly, the Cauchy conditions introduced in Definition $\underline{3}$ correspond exactly 
to the Cauchy conditions for the net $P \mapsto f_{\mu}(P)$.

Proposition 5. Assume that $U, V$ and $W$ are Banach spaces. A function $f: \Omega \rightarrow U$ satisfies the Cauchy criterion for $\mu$-integrability (resp. Cauchy criterion for unconditional $\mu$-integrability over a set $A \subset \Omega$ if and only if $f \in \mathcal{J}(A, \mu, U)$ (resp. $\tilde{J}(A, \mu, U)$ ).

Theorem 6. Assume that $U, V$ and $W$ are Banach spaces, $\mu: \Sigma \rightarrow V$ an integrator and $\tau: U \times V \rightarrow W$ a tensor. The following statements are equivalent for a function $f: \Omega \rightarrow U$ :

1) $f$ is unconditionally $\mu$-integrable over $\mathrm{A} \subset \Omega$.

2) For any injection $\varpi: \Gamma \rightarrow A$, the function $\gamma \mapsto f(\varpi(\gamma))$ is $\eta$-integrable over $\Gamma$, where the integrator $\eta: \varpi^{-1}(\Sigma) \rightarrow V$ is defined by $\eta\left(\varpi^{-1}(E)\right)=\mu(E)$ for all $E \in \Sigma$.

In such a case, one has $\int_{A} \tau(f, \mu)=\int_{\varpi^{-1}(A)} \tau(f \circ \varpi, \mu)$.

Proof. Clearly, $2 \Rightarrow 1$. To see $1 \Rightarrow 2$, suppose $f \in \tilde{\mathcal{J}}(A, \mu, U)$ and let $\varepsilon>0$. Then there exists $P_{0} \in \widetilde{\Pi}(A, \mu)$ such that $\left\|f_{\mu}(R)\right\|_{W}<\varepsilon$ for every $R \in \widetilde{\Pi}(A, \mu), R \cap P_{0}=\emptyset$. Let $\varpi: \Gamma \rightarrow A$ be an injective mapping. We can choose $Q_{0} \in \widetilde{\Pi}(\Gamma, \eta)$ so that $\varpi\left(Q_{0}\right)>P_{0}$. Again, by injectivity of , if $Q \in \widetilde{\Pi}(\Gamma, \eta)$ and $Q \cap Q_{0}=\emptyset$, then $\varpi(Q) \cap \varpi\left(Q_{0}\right)=\varnothing$ and thus $\varpi(Q) \in \widetilde{\Pi}(A, \mu)$ is disjoint from $P_{0}$ and we have $\left\|(f \circ \varpi)_{\eta}(Q)\right\|=$ $\left\|f_{\mu}(\varpi(Q))\right\|<\varepsilon$. Hence, the function $\gamma \mapsto f(\varpi(\gamma))$ is $\eta$-integrable over $\Gamma$. We have established that $1 \Rightarrow$ 2 .

Let $\varepsilon>0$. Choose $Q_{1} \in \widetilde{\Pi}\left(\varpi^{-1}(A) \eta\right)$ such that $\left\|\int_{\varpi^{-1}(A)} \tau(f \circ \varpi, \eta)-(f \circ \varpi)_{\eta}\left(Q_{1}\right)\right\|<\frac{\varepsilon}{3}$. Choose $P_{1} \in \widetilde{\Pi}(A, \mu)$ such that $P_{1}>\varpi\left(Q_{1}\right)$ and $\left\|\int_{A} \tau(f, \mu)-f_{\mu}\left(P_{1}\right)\right\|<\frac{\varepsilon}{3}$. By injectivity, we can choose $Q_{2} \in \widetilde{\Pi}\left(\varpi^{-1}(A), \eta\right) \quad$ such that $\varpi\left(Q_{2}\right)>P_{1} \quad$ and $\quad\left\|\int_{\varpi^{-1}(A)} \tau(f \circ \varpi, \eta)-(f \circ \varpi)_{\eta}\left(Q_{2}\right)\right\|<\frac{\varepsilon}{3}$. Choose $P_{2} \in \widetilde{\Pi}(A, \mu)$ such that $P_{2}>\varpi\left(Q_{2}\right)$ and $\left\|\int_{A} \tau(f, \mu)-f_{\mu}\left(P_{2}\right)\right\|<\frac{\varepsilon}{3}$. Continuing in this way, we construct sequences $n \mapsto P_{n}$ and $n \mapsto Q_{n}$ such that $\varpi\left(Q_{n+1}\right)>P_{n}>\varpi\left(Q_{2}\right)$,

$$
\left\|\int_{\varpi^{-1}(A)} \tau(f \circ \varpi, \eta)-(f \circ \varpi)_{\eta}\left(Q_{n}\right)\right\|<\frac{\varepsilon}{3} \text { and }\left\|\int_{A} \tau(f, \mu)-f_{\mu}\left(P_{2}\right)\right\|<\frac{\varepsilon}{3} .
$$

Now we let $H=\bigcup_{n \in \mathbb{N}} Q_{n}$ and define $\varpi^{\prime}: H \rightarrow A$ by $\varpi^{\prime}(t)=\varpi(t)$. By our hypothesis, the function $t \mapsto f\left(\varpi^{\prime}(t)\right)$ is also $\mu$-integrable. On the other hand, we have

$$
\left\|\int_{\varpi^{-1}(A)} \tau\left(f \circ \varpi^{\prime}, \eta\right)-\left(f \circ \varpi^{\prime}\right)_{\eta}\left(Q_{n}\right)\right\|<\frac{\varepsilon}{3} \text { and }\left\|\int_{A} \tau(f, \mu)-f_{\mu}\left(\varpi^{\prime}\left(Q_{n}\right)\right)\right\|<\frac{\varepsilon}{3} .
$$

We notice that

$$
\left(f \circ \varpi^{\prime}\right)_{\eta}\left(Q_{n}\right)=\sum_{I \in Q_{n}} f\left(\varpi^{\prime}\left(t_{I}\right)\right) \eta(I)=\sum_{\varpi^{\prime}(I) \in \varpi^{\prime}\left(Q_{n}\right)} f\left(\varpi^{\prime}\left(t_{I}\right)\right) \mu\left(\varpi^{\prime}(I)\right)=f_{\mu}\left(\varpi^{\prime}\left(Q_{n}\right)\right) .
$$

By the uniqueness of limit, we have $\int_{A} \tau(f, \mu)=\int_{\varpi^{-1}(A)} \tau(f \circ \varpi, \eta)$ as to be shown. The proof is complete.

Corollary 7. A function $f: \Omega \rightarrow U$ is unconditionally $\mu$-integrable over a set $A$ if and only if it is unconditionally $\mu$-integrable over all subsets of $A$.

\section{Norm and Weak Integrability}

Let $U$ and $V$ be normed spaces, $\Sigma$ a semiring of subsets of a set $A \subset \Omega$. Given a function $f: A \rightarrow U$, and 
$P_{0} \in \Pi(A, \mu)$, we consider $\|f\|_{\mu}(P)=\sum_{I \in P}\left\|f\left(t_{I}\right)\right\|_{U} \mu(I)$ and $u^{*} f_{\mu}(P)=\sum_{I \in P} u^{*}\left(f\left(t_{I}\right)\right) \mu(I)$ for every $u^{*} \in$ $U^{*}$. We then say that $f: A \rightarrow U$ is norm $\mu$-integrable (resp. weakly $\mu$-integrable) if $\int_{A}\|f\| d \mu:=\lim _{P}\|f\|_{\mu}(P)$ (resp. for every $u^{*} \in U^{*}, \int_{A} u^{*} f d \mu:=\lim _{P} u^{*} f_{\mu}(P)$ ) represents a scalar.

We denote by $I^{1}(A, \mu, U)$ (resp. $I^{w}(A, \mu, U)$ ) the space of classes of norm (resp. weak) $\mu$-integrable functions. It is immediately seen that $I^{1}(A, \mu, U) \subset I^{w}(A, \mu, U)$. For example, if $\mu$ is a non-negative measure on a $\sigma$-algebra containing the Borel $\sigma$-algebra of a given set $\Omega$, and $U$ a Banach space, then $L^{1}(A, \mu, U) \subset I^{1}(A, \mu, U)$. We also see that Pettis $\mu$-integrable functions are weakly $\mu$-integrable [2] .

Clearly, a function $f$ is in $I^{1}(A, \mu, U)$ if and only if the scalar function $\|f\|: A \rightarrow \mathbb{R}$ is in $I(A, \mu, \mathbb{R})$. More generally, for $0<p<\infty$, we also define $I^{p}(A, \mu, U):=\left\{f: A \rightarrow U:\|f\|^{p} \in I(A, \mu, \mathbb{R})\right\}$.

For example, it is easily seen that $L^{p}(A, \mu, U) \subsetneq I^{P}(A, \mu, U)$ for $0<p<\infty$. As in Theorem $\underline{1}, I^{P}(A, \mu, U)$ is a Banach space whenever $U$ is complete. Moreover, if $1<p, q<\infty$ are such that $1 / p+1 / q=1$, then the Riesz Representation Theorem states that whenever the Banach space $U^{*}$ has the Radon-Nikodym property [2], then $\left[L^{p}(A, \mu, U)\right]^{*} \cong L^{q}\left(A, \mu, U^{*}\right)$. Such a restriction is not needed for the $I^{P}(A, \mu, U)$ spaces.

Theorem 8. Let $U, V$ and $W$ be Banach spaces, $\mu: \Sigma \rightarrow V$ an integrator and $\tau: U \times V \rightarrow W$. If $1<p, q<\infty$ are such that $1 / p+1 / q=1$, then $\left[I^{p}(A, \Sigma, \mu, U)\right]^{*} \cong I^{q}\left(A, \Sigma, \mu, U^{*}\right)$.

The reader is referred to [11] for the proof of the above theorem and more detailed presentation on results related to the Riesz Representation Theorem.

\section{References}

[1] Bartle, R. G. (2001). A Modern Theory of Integration, Graduate Studies in Math. AMS, Providence, RI.

[2] Diestel, J., \& Uhl, J. J., (1977). Vector Measures, AMS Mathematical Survey 15. Providence, RI.

[3] Dinculeanu, N. (2000). Vector Integration and Stochastic Integration in Banach Spaces. Wiley \& Sons Inc.

[4] Dunford, N., \& Schwartz, J. (1958) Linear Operators, Part I, General Theory. Wiley Interscience, New York

[5] The Bartle-Dunford-Schwartz integral. (2008). Monografie Matematyczne (instytut Matematyczny Polskiej Akademii Nauk), vol. 69. Birkhauser basel.

[6] Rodriguez, J. (2006). On integration of vector functions with respect to vector measures, Czechoslovak Mathematical Journal, 56(131), 805-825.

[7] Robdera, M. A., \& Kagiso D. N. (2017). Fundamental theorem of calculus in topological vector spaces. Journal of Mathematics Extension, 11(2), 93-110.

[8] Robdera, M. A. (2016). Extensions of the Lusin's theorem, the Severini-Egorov's theorem and the Riesz subsequence theorems. Asian Research J. Math, 1(4), 1-9.

[9] Robdera, M. A. (2015). On the Riesz representation theorem and integral operators. Quaestiones Mathematicae, 1-15.

[10] Robdera, M. A. (2015). On natural characterization of tensor integrability. British J. Math. \& Comp. Sci., $8(6), 458-469$.

[11] Robdera, M. A. (2014). Tensor integral: a new comprehensive approach to integration theory. British J. Math. \& Comp. Sci., 4(22), 3236-3244.

[12] Robdera, M. A., \& Kagiso, D. N. (2013). On the differentiability of vector valued additive set functions. Adv. in Pure Math., 3, 653-659.

[13] Kurtz, D. S., \& Swartz, C. (2004). Theories of Integration. World Sci. Pub. Co. Pte. Ltd., Singapore.

[14] McShane, E. J. (1952). Partial orderings and moore-smith limits. Amer. Math, 59, 1-11.

[15] Diestel, J., Fourie, J., \& Swart, J. (2008). The Metric Theory of Tensor Products. AMS Providence, RI. 


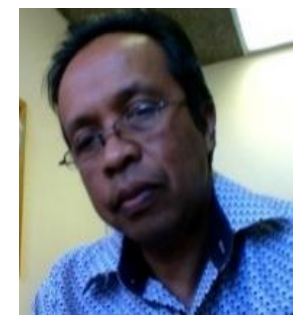

Mangatiana A. Robdera received his Ph.D degree in pure mathematics at the University of Missouri (USA) in 1996. He specializes in functional analysis. His research interests are in the areas of Geometry of Banach spaces, Vector Measures and Integration,

Mangatiana has over twenty years of teaching experience at the tertiary education level: University of Madagascar-Antananarivo, University of Missouri-Columbia (USA), William Paterson Univesrsity, NJ (USA), Eastern Mediterranean University, Northern Cyprus, Al-Akhawayn Univesity, Morocco. He currently holds an associate professor position at the University of Botswana in Gaborone. He has published several research papers at various renowned international peer reviewed journals such as Journal of Mathematical Analysis and Applications, Glasgow Journal of Mathematics, Quaestiones Mathematicae, Advanced in Pure Mathematics, International Journal of Modeling and Optimization. He also published two textbooks.

Prof. Robdera is a member of the American Mathematical Society, the Southern African Mathematical Science Association, la Société de Mathématiques de Madagascar. In addition, Prof. Robdera is a member of the editorial board of Journal of Advances in Mathematics and Computer Science, the editorial board of Pure and Applied Mathematics Journal, and avail himself as a reviewer for the American Mathematical Society. 